\title{
THE NATURAL APPROACH OF \\ WILKER-CUSA-HUYGENS INEQUALITIES
}

\section{CRISTINEL MORTICI}

Abstract. The aim of this paper is to provide a natural approach of Wilker-Cusa-Huygens inequalities. This new approach permits us to give new proofs then to refine much these inequalities and we are convinced that it is suitable to establish many other similar inequalities. To attain these purposes, computer softwares such as Maple are of great importance throughout this work.

Mathematics subject classification (2010): 26D05; 26D15; 33B10.

Keywords and phrases: trigonometric inequalities, power series.

\section{Introduction}

The starting point of this paper is the following double inequality

$$
(\cos x)^{1 / 3}<\frac{\sin x}{x}<\frac{2+\cos x}{3} \quad\left(0<x<\frac{\pi}{2}\right),
$$

which has attracted the attention of many authors in the recent past.

The left-hand side inequality (1.1) first appeared in [4, p. 238], while the righthand side inequality (1.1) was first mentioned by the German philosopher and theologian Nicolaus de Cusa (1401-1464). A rigorous proof of Cusa's inequality was given by Huygens [3], who used (1.1) to estimate the number $\pi$. Further interesting historical facts about the inequalities (1.1) can be found in [7].

Cusa proved (1.1) by using certain geometrical constructions, but in modern times, such inequalities were proved using the variation of some functions and their derivatives.

We give in this paper a new method for proving trigonometric inequalities of type (1.1), using the expansions

$$
\sin x=\sum_{k=0}^{\infty} \frac{(-1)^{k}}{(2 k+1) !} x^{2 k+1}, \quad \cos x=\sum_{k=0}^{\infty} \frac{(-1)^{k}}{(2 k) !} x^{2 k} .
$$

More precisely, we use the following inequalities for $x \in(0, \pi / 2)$ and non-negative integers $m$ and $n$,

$$
\begin{gathered}
a(x, 2 n+1)<\sin x<a(x, 2 n) \\
b(x, 2 m+1)<\cos x<b(x, 2 m),
\end{gathered}
$$


which are a direct consequence of the classical Taylor's theorem, where

$$
a(x, p)=\sum_{k=0}^{p} \frac{(-1)^{k}}{(2 k+1) !} x^{2 k+1}, \quad b(x, q)=\sum_{k=0}^{q} \frac{(-1)^{k}}{(2 k) !} x^{2 k} .
$$

The first inequality (1.1) is equivalent to

$$
\cos x-\left(\frac{\sin x}{x}\right)^{3}<0
$$

but we improve it by giving the following accurate estimates

$$
-\frac{x^{4}}{15}<\cos x-\left(\frac{\sin x}{x}\right)^{3}<-\frac{x^{4}}{15}+\frac{23 x^{6}}{1890} \quad\left(0<x<\frac{\pi}{2}\right),
$$

then we refine the second inequality (1.1) to

$$
-\frac{1}{180} x^{4}<\frac{\sin x}{x}-\frac{\cos x+2}{3}<-\frac{1}{180} x^{4}+\frac{1}{3780} x^{6} \quad\left(0<x<\frac{\pi}{2}\right) .
$$

The following nice inequality due to Huygens [3]

$$
2 \frac{\sin x}{x}+\frac{\tan x}{x}>3 \quad\left(0<x<\frac{\pi}{2}\right)
$$

is a consequence of (1.1), see [1]. We show in the next section the inequality

$$
3+\left(\frac{3 x^{4}}{20}-\frac{3 x^{6}}{140}\right) \frac{1}{\cos x}<2 \frac{\sin x}{x}+\frac{\tan x}{x}<3+\frac{3 x^{4}}{20 \cos x} \quad\left(0<x<\frac{\pi}{2}\right),
$$

which improves much (1.4).

The following inequality

$$
\left(\frac{\sin x}{x}\right)^{2}+\frac{\tan x}{x}>2 \quad\left(0<x<\frac{\pi}{2}\right)
$$

due to Wilker [9] was intensively studied by many authors, e.g. [2], [6], [8], [10]-[13]. Related to (1.5), we prove that

$$
2+\left(\frac{8 x^{4}}{45}-\frac{4 x^{6}}{105}\right) \frac{1}{\cos x}<\left(\frac{\sin x}{x}\right)^{2}+\frac{\tan x}{x}<2+\frac{8 x^{4}}{45 \cos x} \quad\left(0<x<\frac{\pi}{2}\right) .
$$

$\mathrm{Wu}$ and Srivastava [10, Lemma 3] proved the following dual inequality

$$
\left(\frac{x}{\sin x}\right)^{2}+\frac{x}{\tan x}>2 \quad\left(0<x<\frac{\pi}{2}\right),
$$

but we show in this paper the following refinement

$$
\left(\frac{x}{\sin x}\right)^{2}+\frac{x}{\tan x}>2+\frac{2}{45} x^{4} \quad\left(0<x<\frac{\pi}{2}\right) .
$$


Then we refine a result of Neuman and Sandor [5, Theorem 2.3] who showed

$$
3 \frac{x}{\sin x}+\cos x>4 \quad\left(0<x<\frac{\pi}{2}\right),
$$

establishing that

$$
3 \frac{x}{\sin x}+\cos x>4+\frac{1}{10} x^{4}+\frac{1}{210} x^{6} \quad\left(0<x<\frac{\pi}{2}\right) .
$$

\section{The results}

In this section we prove the announced inequalities, using as main tool the estimates (1.2)-(1.3).

THEOREM 1. For every $0<x<\pi / 2$, we have

$$
-\frac{x^{4}}{15}<\cos x-\left(\frac{\sin x}{x}\right)^{3}<-\frac{x^{4}}{15}+\frac{23 x^{6}}{1890} .
$$

Proof. By (1.2)-(1.3) inequalities, we get

$$
\cos x-\left(\frac{\sin x}{x}\right)^{3}+\frac{x^{4}}{15}>b(x, 3)-\left(\frac{a(x, 2)}{x}\right)^{3}+\frac{x^{4}}{15}=\frac{x^{6} P\left(x^{2}\right)}{1728000}>0,
$$

where $P(t)=20000-1560 t+60 t^{2}-t^{3}$. The positivity of $P$ on $\left(0, \pi^{2} / 4\right)$ follows from the fact that $P$ is strictly decreasing, with $P\left(\pi^{2} / 4\right)>0$. Then

$$
\begin{aligned}
\cos x-\left(\frac{\sin x}{x}\right)^{3}+\frac{x^{4}}{15}-\frac{23 x^{6}}{1890} & <b(x, 4)-\left(\frac{a(x, 3)}{x}\right)^{3}+\frac{x^{4}}{15}-\frac{23}{1890} x^{6} \\
& =-\frac{x^{8} Q\left(x^{2}\right)}{128024064000}<0,
\end{aligned}
$$

where $Q(t)=137803680-7832160 t+300888 t^{2}-7812 t^{3}+126 t^{4}-t^{5}$. We have

$$
Q^{\prime}(t)=601776 t-23436 t^{2}+504 t^{3}-5 t^{4}-7832160
$$

and $Q^{\prime}(3-w)=-474228 w-19170 w^{2}-444 w^{3}-5 w^{4}-6224553$. As a consequence, $Q(t)>0$, for every $t \in\left(0, \pi^{2} / 4\right)$, since $Q$ is strictly decreasing on $[0,3]$, with $Q\left(\pi^{2} / 4\right)$ $>0$.

THEOREM 2. For every $0<x<\pi / 2$, we have

$$
-\frac{1}{180} x^{4}<\frac{\sin x}{x}-\frac{\cos x+2}{3}<-\frac{1}{180} x^{4}+\frac{1}{3780} x^{6} .
$$


Proof. By (1.2)-(1.3) inequalities, we get

$$
\frac{\sin x}{x}-\frac{\cos x+2}{3}+\frac{1}{180} x^{4}>\frac{a(x, 3)}{x}-\frac{b(x, 4)+2}{3}+\frac{1}{180} x^{4}=\frac{x^{6}\left(32-x^{2}\right)}{120960}>0 .
$$

Then

$$
\begin{aligned}
\frac{\sin x}{x}-\frac{\cos x+2}{3}+\frac{1}{180} x^{4}-\frac{1}{3780} x^{6} & <\frac{a(x, 4)}{x}-\frac{b(x, 5)+2}{3}+\frac{1}{180} x^{4}-\frac{1}{3780} x^{6} \\
& =-\frac{x^{8}\left(60-x^{2}\right)}{10886400}<0 .
\end{aligned}
$$

THEOREM 3. For every $0<x<\pi / 2$, we have

$$
3+\left(\frac{3 x^{4}}{20}-\frac{3 x^{6}}{140}\right) \frac{1}{\cos x}<2 \frac{\sin x}{x}+\frac{\tan x}{x}<3+\frac{3 x^{4}}{20 \cos x} .
$$

Proof. By multiplying by $\cos x$, we obtain the equivalent form

$$
3 \cos x+\frac{3 x^{4}}{20}-\frac{3 x^{6}}{140}<\frac{\sin 2 x}{x}+\frac{\sin x}{x}<3 \cos x+\frac{3 x^{4}}{20} .
$$

By (1.2)-(1.3) inequalities, we get

$$
\begin{aligned}
\frac{\sin 2 x}{x}+\frac{\sin x}{x}-3 \cos x-\frac{3 x^{4}}{20}+\frac{3 x^{6}}{140} & >\frac{a(2 x, 5)}{x}+\frac{a(x, 5)}{x}-3 b(x, 4)-\frac{3 x^{4}}{20}+\frac{3 x^{6}}{140} \\
& =\frac{x^{8}\left(17820-683 x^{2}\right)}{13305600}>0 .
\end{aligned}
$$

Then

$$
\begin{aligned}
\frac{\sin 2 x}{x}+\frac{\sin x}{x}-3 \cos x-\frac{3 x^{4}}{20} & <\frac{a(2 x, 4)}{x}+\frac{a(x, 2)}{x}-3 b(x, 3)-\frac{3 x^{4}}{20} \\
& =-\frac{x^{6}\left(963-64 x^{2}\right)}{45360}<0 .
\end{aligned}
$$

THEOREM 4. For every $0<x<\pi / 2$, we have

$$
2+\left(\frac{8 x^{4}}{45}-\frac{4 x^{6}}{105}\right) \frac{1}{\cos x}<\left(\frac{\sin x}{x}\right)^{2}+\frac{\tan x}{x}<2+\frac{8 x^{4}}{45 \cos x}
$$

Proof. By multiplying by $\cos x$, we obtain the equivalent form

$$
2 \cos x+\frac{8 x^{4}}{45}-\frac{4 x^{6}}{105}<\left(\frac{\sin x}{x}\right)^{2} \cos x+\frac{\sin x}{x}<2 \cos x+\frac{8 x^{4}}{45} .
$$


By (1.2)-(1.3) inequalities, we get

$$
\begin{aligned}
& \left(\frac{\sin x}{x}\right)^{2} \cos x+\frac{\sin x}{x}-2 \cos x-\frac{8 x^{4}}{45} \\
< & \left(\frac{a(x, 2)}{x}\right)^{2} b(x, 2)+\frac{a(x, 2)}{x}-2 b(x, 3)-\frac{8 x^{4}}{45} \\
= & -\frac{x^{6} R\left(x^{2}\right)}{345600}<0,
\end{aligned}
$$

where $R(t)=12480-1144 t+52 t^{2}-t^{3}$. We have $R(t)>0$, for every $t \in\left(0, \pi^{2} / 4\right)$, since $R$ is strictly decreasing with $R\left(\pi^{2} / 4\right)>0$.

Then

$$
\begin{aligned}
& \left(\frac{\sin x}{x}\right)^{2} \cos x+\frac{\sin x}{x}-2 \cos x-\frac{8 x^{4}}{45}+\frac{4 x^{6}}{105} \\
> & \left(\frac{a(x, 3)}{x}\right)^{2} b(x, 3)+\frac{a(x, 3)}{x}-2 b(x, 4)-\left(\frac{8 x^{4}}{45}-\frac{4 x^{6}}{105}\right) \\
= & \frac{x^{8} S\left(x^{2}\right)}{18289152000}>0,
\end{aligned}
$$

where $S(t)=72938880-4848480 t+214920 t^{2}-6324 t^{3}+114 t^{4}-t^{5}$ is strictly decreasing on $\left(0, \pi^{2} / 4\right)$, with $S\left(\pi^{2} / 4\right)>0$.

THEOREM 5. For every $0<x<\pi / 2$, we have

$$
\left(\frac{x}{\sin x}\right)^{2}+\frac{x}{\tan x}>2+\frac{2}{45} x^{4}
$$

Proof. By (1.2)-(1.3) inequalities, we get

$$
\begin{aligned}
\left(\frac{x}{\sin x}\right)^{2}+\frac{x \cos x}{\sin x}-\left(2+\frac{2}{45} x^{4}\right) & >\left(\frac{x}{a(x, 2)}\right)^{2}+\frac{x b(x, 3)}{a(x, 2)}-\left(2+\frac{2}{45} x^{4}\right) \\
& =\frac{Z\left(x^{2}\right)}{90\left(x^{4}-20 x^{2}+120\right)^{2}}>0,
\end{aligned}
$$

where $Z(t)=10200-1990 t+145 t^{2}-4 t^{3}$ is strictly positive on $\left(0, \pi^{2} / 4\right)$, since $Z$ is strictly decreasing with $Z\left(\pi^{2} / 4\right)>0$.

THEOREM 6. For every $0<x<\pi / 2$, we have

$$
3 \frac{x}{\sin x}+\cos x>4+\frac{1}{10} x^{4}+\frac{1}{210} x^{6} .
$$


Proof. By (1.2)-(1.3) inequalities, we get

$$
\begin{aligned}
3 \frac{x}{\sin x}+\cos x-\left(4+\frac{1}{10} x^{4}+\frac{1}{210} x^{6}\right) & >3 \frac{x}{a(x, 4)}+b(x, 5)-\left(4+\frac{1}{10} x^{4}+\frac{1}{210} x^{6}\right) \\
& =\frac{x^{8} V\left(x^{2}\right)}{3628800 U\left(x^{2}\right)}>0
\end{aligned}
$$

where

$$
U(t)=3024 t^{2}-60480 t-72 t^{3}+t^{4}+362880
$$

and

$$
V(t)=1728000 t^{2}-59875200 t-31824 t^{3}+162 t^{4}-t^{5}+862202880 .
$$

Finally remark that $U(t)>0$ and $V(t)>0$, for every $t \leqslant 3$, since the polynomials $U(3-w)$ and $V(3-w)$ have all coefficients positive.

We are convinced that our new approach is suitable for proving and establishing many other inequalities also involving hyperbolic functions. As an example, we mention the following result [5, Corollary 2.3]

$$
2 \frac{\sinh x}{x}+\frac{\tanh x}{x}>3 \quad(x>0) .
$$

Remark that this inequality can be improved to

$$
2 \frac{\sinh x}{x}+\frac{\tanh x}{x}>3+\frac{3}{20} x^{4}-\frac{3}{56} x^{6} \quad(x>0),
$$

using the following estimates

$$
\sinh x>x+\frac{1}{6} x^{3}+\frac{1}{120} x^{5}+\frac{1}{5040} x^{7}, \quad \tanh x>x-\frac{1}{3} x^{3}+\frac{2}{15} x^{5}-\frac{17}{315} x^{7} .
$$

arising from the corresponding expansions in power series of $x$.

\section{REFERENCES}

[1] A. BARICZ AND J. SÁNDOR, Extensions of generalized Wilker inequality to Bessel functions, J. Math. Inequal., 2, 3 (2008) 397-406.

[2] B.-N. GuO, B.-M. QIAO, F. QI, AND W. LI, On new proofs of Wilker inequalities involving trigonometric functions, Math. Inequal. Appl., 6, 1 (2003), 19-22.

[3] C. Huygens, Oeuvres completes, publiées par la Société hollandaise des science, Haga, 1888-1940.

[4] D. S. Mitrinović, Analytic Inequalities, Springer-Verlag, Berlin, 1970.

[5] E. NEUMAN AND J. SÁNDOR, On some inequalities involving trigonometric and hyperbolic functions with emphasis on the Cusa-Huygens, Wilker, and Huygens inequalities, Math. Inequal. Appl., 13, 4 (2010), 715-723.

[6] I. PINELIS, L'Hospital rules of monotonicity and Wilker-Anglesio inequality, Amer. Math. Monthly, 111 (2004), 905-909.

[7] J. SÁndor And M. BEnCZE, On Huygens' trigonometric inequality, RGMIA Res. Rep. Collection 8, 3 (2005) Article 14. 
[8] J. S. Sumner, A. A. Jagers, M. Vowe, And J. Anglesio, Inequalities involving trigonometric functions, Amer. Math. Monthly, 98 (1991), 264-267.

[9] J. B. Wilker, Problem E 3306, Amer. Math. Monthly, 96 (1989) 55.

[10] S.-H. WU AND H. M. SRIVAstaVA, A weighted and exponential generalization of Wilker's inequality and its applications, Integral Transforms and Spec. Funct., 18, 8 (2007), 525-535.

[11] S.-H. WU AND H. M. SRIVASTAVA, A further refinement of Wilker's inequality, Integral Transforms and Spec. Funct., 19, 10 (2008), 757-765.

[12] L. ZHU, A new simple proof of Wilker's inequality, Math. Inequal. Appl., 8, 4 (2005), 749-750.

[13] L. ZHU, On Wilker-type inequalities, Math. Inequal. Appl., 10, 4 (2007), 727-731. 